\title{
IMPLEMENTING THE ANALYTIC HIERARCHY PROCESS AS A STANDARD METHOD FOR MULTI-CRITERIA DECISION MAKING IN CORPORATE ENTERPRISES - A NEW AHP EXCEL TEMPLATE WITH MULTIPLE INPUTS
}

\author{
Klaus D. Goepel \\ No 10 Changi Business Park Central 2 \\ Hansapoint@CBP \#06-01/08 \\ Singapore 486030 \\ E-mail: drklaus@bpmsg.com
}

\begin{abstract}
Though the analytic hierarchy process (AHP) is universal and powerful in its application, it is still simple enough to be implemented in a spreadsheet program like MS Excel. In this paper the author describes the development of a general, freely available AHP Excel template, allowing for multiple inputs with individual and consolidated output for decision makers. After an explanation of the template's structure, realization and limitations, its practical use is illustrated with actual examples. They range from the determination of weights for key performance indicators in business performance management, over the ranking of growth strategies for a company, to the selection of leadership competencies for a leadership development program. Experiences and challenges in the implementation and application of AHP will be highlighted. For the analysis of the group judgments within the projects, a new consensus indicator is introduced. It is based on the concept of diversity using Shannon entropy. Partitioning into two independent components allows finding clusters of high consensus within groups of decision makers.
\end{abstract}

Keywords: spreadsheet, template, group decision making, entropy, consensus indicator

\section{Introduction}

Although from a rational and logical point of view there are many arguments to use analytic methods and supporting tools for complex decision making in a corporate environment, in practice obstacles and hurdles have to be overcome when introducing a multi-criteria decision making method (MCDM) as a new tool in the decision making process. Decision making processes in a corporate environment are often a result of relationships, formal or informal discussions, networking and political interests. Any changes will at first be seen critically and observed skeptically, before advantages are recognized and accepted. In the following a few points are highlighted from the author's view and experience:

- The method must be explainable and understandable for managers and decision makers, without the need to go into technical or scientific details. Once you start talking about matrices, eigenvalues or rank reversal, it will be difficult to get further attention or acceptance.

- Decision making - even for complex decision problems - also works without MCDM. Any investment in a software package will require persistence and convincing arguments, as it applies for all other investments, where the benefit cannot be seen immediately.

- The procedure of getting inputs for the decision problem and selected method (i.e. ratings, evaluations, questionnaires) must not exceed the effort and time required without the use of the method. It will be very convincing, if a decision problem can be solved in an even shorter time.

Based on this the author started to introduce AHP as a new MCDM, starting with a few smaller projects. A review of the main developments in AHP is given by (Ishizaka, Labib 2011). As AHP is still implementable in a spreadsheet program, and to avoid an initial investment in commercial software, an AHP template was developed and realized using Excel. 


\section{AHP Spreadsheet Template}

\subsection{Requirements}

Before the actual implementation a list of requirements had to be considered. Beside easy-to-use, working without macros and not relying on external links to other workbooks, the template should be flexible in the number of criteria, the number of participants and level of accepted inconsistency in the matrix. In addition each questionnaire should fit on one page for printing and manual completion.

\subsection{Realization}

As a result a flexible spreadsheet template with the following features was realized, which was then used in all AHP projects.

- The workbook consists of 10 (or more) input worksheets for pair-wise comparisons, a sheet for the consolidation of all judgments, a summary sheet to display the result, a sheet with reference tables (random index, limits for geometric consistency index GCI, judgment scales) and a sheet for solving the eigenvalue problem when using the eigenvector method (EVM)

- Within the input worksheets (questionnaires), priorities are calculated using the row geometric mean method (RGMM).

- Two consistency indices (the consistency ratio CR and the geometric consistency index GCI) are calculated. The level of consistency needed $(\alpha) \square$ is implemented as a variable input field, and can be set between zero and one.

- If CR exceeds $\alpha$, the top 3 inconsistent pair-wise comparisons are highlighted, to allow the participants an adjustment of their judgments.

- Final priorities are shown in a summary sheet; their calculation is based on the eigenvector method (EVM). For the solution of the eigenvalue problem the power method algorithm (e.g. Larsen, 2013) is applied with a fixed number of 12 iterations.

- Different judgment scales are implemented.

- Either individual participants, or an aggregation of individual judgments (AIJ) based on the geometric mean of all participants' judgments (Aull-Hyde et al., 2006), can be selected.

The implementation as a spreadsheet template also resulted in some limitations; the template does not include the hierarchy of the decision problem and the final aggregation of weights, i.e. it is only suitable for finding the weights in each category or sub-category. Another limitation is the lack of sensitivity analysis of the final result.

\section{Experiences in the practical application of AHP}

We used this template in a number of actual projects for a multi-national corporation (MNC). The company has its headquarters in Germany, and is globally represented with subsidiaries in Europe, the Americas and the Asia/Pacific region. The main objectives for introducing AHP to this corporation were on the one hand, to make the decision process more structured and transparent, on the other hand to make a tool available for group decisions using mathematical aggregation, especially for decisions with a larger number of stakeholders at different locations.

\subsection{AHP Projects}

Table 1 gives an overview on the AHP projects referenced in this paper. All projects were multinational, involving several countries and nationalities. Project 1 and 2 were smaller projects. The objective here was to find consensus on the weights of performance indicators (PI); no complex hierarchy was necessary. Project 3 was related to the final selection of PI out of a set of preselected PI in the development of a dashboard, to measure the performance of sales subsidiaries. An initial set of 
10 indicators in the perspectives market, finance and productivity was evaluated by 6 experts from sales, sales controlling and finance departments. As a result, based on the consolidated priorities, the original set of 10 performance indicators could be reduced to 6 key performance indicators (KPI), fulfilling the information needs of all stakeholders.

Table 1. AHP projects described in this paper

\begin{tabular}{|l|l|l|l|l|l|l|}
\hline No & AHP Project Name & $\begin{array}{l}\text { No of } \\
\text { cat. }\end{array}$ & $\begin{array}{l}\text { No of } \\
\text { Criteria }\end{array}$ & DM & $\begin{array}{l}\text { Coun- } \\
\text { tries }\end{array}$ & Outcome/Remark \\
\hline 1 & Service KPIs & 1 & 5 & 2 & 2 & Combined performance Index \\
\hline 2 & Customer Hotline KPIs & 1 & 5 & 5 & 4 & MBO, customer survey form \\
\hline 3 & Company Performance & 3 & $4+4+2$ & 6 & 2 & $\begin{array}{l}\text { Development of a company } \\
\text { performance dashboard }\end{array}$ \\
\hline 4 & Strategy & 3 & $6+6+6$ & 65 & 14 & 5-year strategy and reorganizations \\
\hline 5 & Leadership Values & 1 & 7 & 4 & 2 & Leadership assessment \\
\hline
\end{tabular}

After collecting experiences in the first practical applications of AHP with these smaller projects, the more complex project 4 was started. The objective here was to identify, prioritize and align strategies for growth of the company beyond normal market growth within the next 3 to 5 years, and translate them into measurable targets using the balanced scorecard.

The problem was first approached using the concept of strategy maps (Kaplan, Norton, 2004). After the development of the basic strategies in the strategic map, different strategic approaches were proposed for the perspectives finance, customer (customer value proposition, sales), internal (organization), and learning and growth (human capital).

The project could then be easily structured hierarchically (Figure 1), and managers, experts and other stakeholders were identified to do an evaluation in their categories or sub-categories. To reduce the number of questionnaires, the 6 criteria shown under perspective organization \& capital were combined into one AHP questionnaire; under the category customer value propositions two questionnaires with 6 criteria were sent out, one for each business field. In total 65 managers and decision makers (DM) from 14 countries were involved in this project.

Most of the questionnaires had to be sent out by e-mail with some basic information about AHP and an extensive description of how to fill out the pair-wise comparison and to use the scale. This would be a typical situation in many MNCs as often there is no opportunity to get all stakeholders together in a meeting when the project extends to several countries or subsidiaries.

Project 5 was conducted to find the importance of seven leadership values for a leadership assessment and leadership development program. 


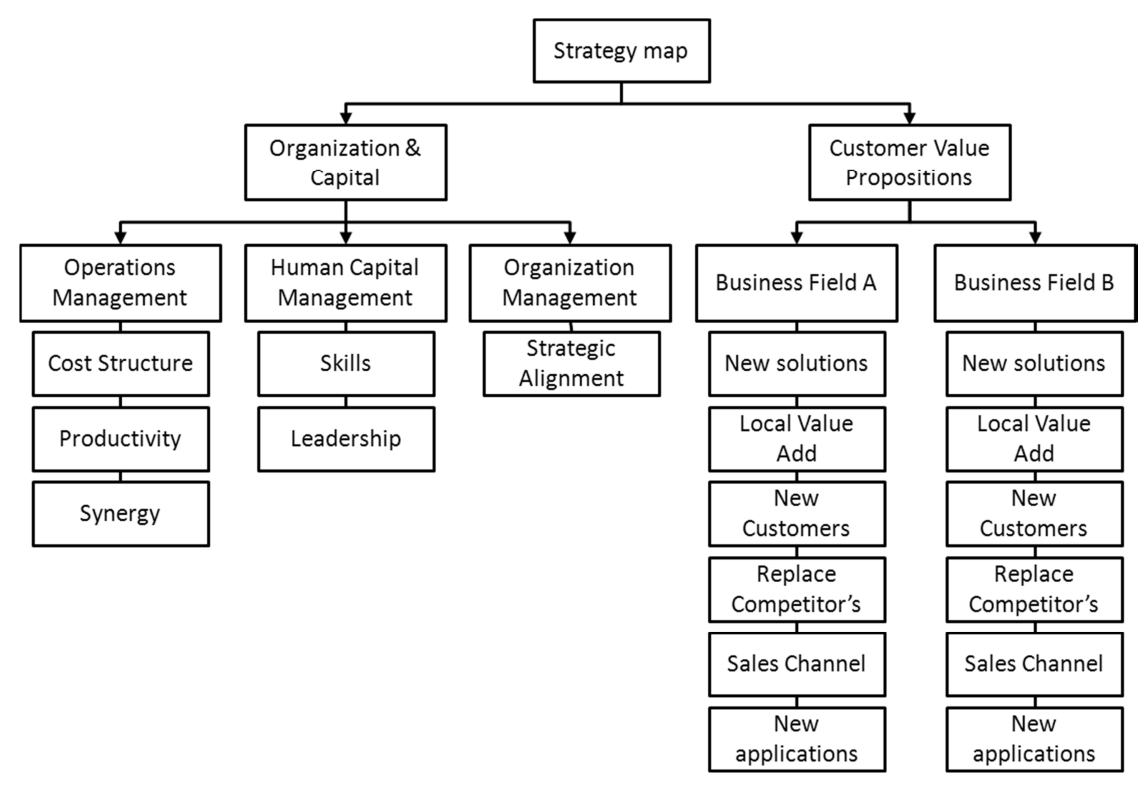

Figure 1. Hierarchical structure of the strategy project handled with AHP as described in the text

\section{Observations and Experiences}

\subsection{Hierarchical Structuring}

Structuring the decision problem in a hierarchy was not a difficult issue in any of our projects; often the nature of a problem already covers different aspects or perspectives, which can be used in a first approach to further breakdown in categories and sub-categories. From our practical experience each category should have at least 4 , but not more than 7 to 10 sub-categories. Three criteria can easily be weighted without using AHP; more than 10 sub-categories require over 45 pair-wise comparisons; this gets easily too complex and confusing for DM. In the case of the strategy project (project 4) it was also helpful to have - as a supporting tool - the concept of strategy maps available. This simplified the hierarchical structuring significantly. During the feedback sessions with DM in all our projects the structuring was never a major point of discussions; indeed AHP follows the intuitive way in which managers solve problems.

\subsection{Response to the AHP questionnaire}

After sending out the questionnaires, the majority of responses came back completed correctly, without many requests for further clarifications about the completion procedure, the pair-wise comparison or the AHP scale. The background information and instructions given seemed to be quite understandable and clear.

\subsection{Consistency}

In AHP priorities are derived from consistent or near consistent matrices. A measure of consistency is the consistency index $C I$. From this a consistency ratio $C R=C I / R I$ is derived, using a randomized index $R I$, the average $C I$ for randomly filled matrices. Other consistency measures have been proposed, an overview can be found for example in (Bozóki, Rapcsák, 2007). For some consistency measures proportionality between them was proved. (Brunelli et al., 2011). We used either Saaty's proposed CR, or a modified CR, as proposed by (Alonso, Lamata 2006) for the eigenvector method, and the geometric consistency index GCI for RGMM. We also introduced $\alpha$ as a level of consistency needed to adapt the cut-off value $(\mathrm{CR}<\alpha)$, when to declare the matrix inconsistent.

The question we then had to answer was: what is the right level of $\alpha$ in relation to our specific projects? Saaty's rule of thumb is to accept only judgment matrices with $\mathrm{CR}<0.1$. Figure 2 shows the 
distribution of CR values for all (80) participants in our projects. Median value is $\mathrm{CR}=0.16$ (linear AHP 1-9 scale), $80^{\text {th }}$-percentile is at $\mathrm{CR}=0.36$. The ten-percent rule is obviously too strict for our practical applications, and would have resulted in rejection and revision of the majority of questionnaires.

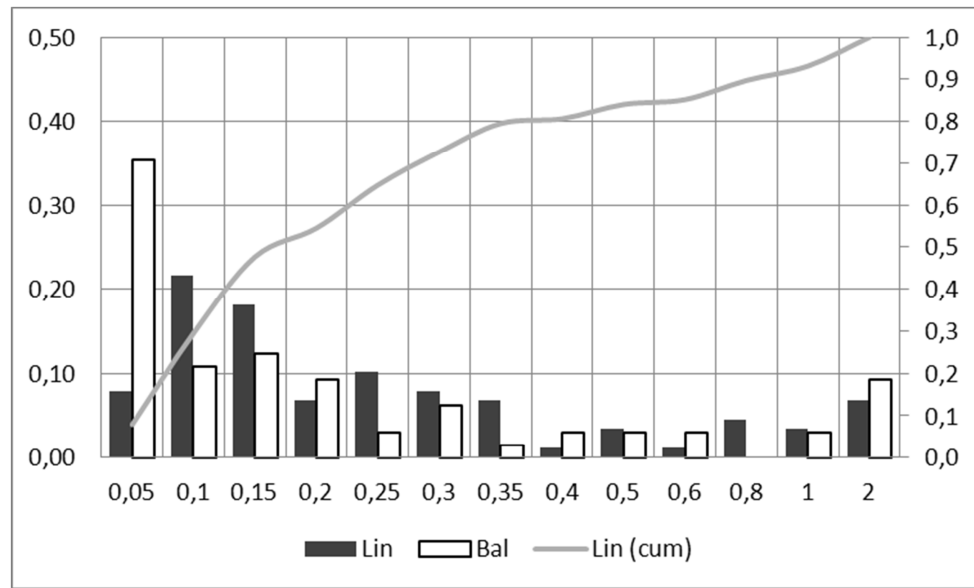

Figure 2. Relative frequency of consistency ratio CR for all participants

As a revision of so many inputs was not practicable, we defined for project 4 a threshold of $\alpha=0.3$ as limit for CR. To gain more confidence in the results, we used, in addition to the linear scale, the balanced judgment scale (Salo, Hämäläinen, 1997). For the balanced scale the median value is 0.06, $80^{\text {th }}$-percentile is below 0.2. As the objective was to identify the 3 top strategies for individual countries, the exact percentage of weights did not have a high impact. All calculated final priorities were also reconfirmed by the DM.

As a summary we can say that the requirement of consistency is the most critical issue in the practical application of AHP. The use of the balanced scale improves consistency, but it would be most helpful to have well defined, theoretically founded cut-off limits, independent from scales and priority derivation methods. (Siraj, 2011) could be a basis for further studies.

\section{Group decision making}

One of the main reasons to use AHP in our projects was to come to group decisions, for example to select key performance indicators and define their weights in a performance dashboard (project 1 and 2 ), or to agree on common strategies for future growth of the company. Our experience in all projects was a high acceptance of the aggregated group results by all stakeholders. Helpful is the fact that AHP - as a mathematical method - is seen to be "objective", and decision makers appreciate that their judgments are reflected in the final outcome.

On the other hand, we also wanted to get a picture about the actual consensus of DM in the different projects. Dispersion of group judgment in the context of AHP was already investigated, e.g. in (Saaty, Vargas, 2005). We used a different approach, adapting the concept of diversity, as it is established and applied in biology and ecology (Jost, 2006). Species richness and relative abundance correspond to the number of criteria and calculated priorities in our application. Diversity can be characterized by several diversity indexes, for example Shannon entropy, Gini-Simpson index or Rényi entropies. (Jost, 2006) provided a unified mathematical framework for computation and use of number equivalents of classical diversity measures, known as "true diversity" or Hill numbers (Hill, 1973).

\subsection{Shannon Entropy $\boldsymbol{H}$}

For our application we selected the Shannon entropy H (Shannon, 1949) as a diversity index for the distribution of priorities among criteria; it can be written as 


$$
H=-\sum_{i=1}^{N} p_{i} \ln p_{i}
$$

With $p_{i}$ calculated priorities for criteria $i=1$ to $N$. True diversity of order one $(D)$ is then given by

$$
D=\exp H
$$

Practically $H$ can be interpreted as a measure of the evenness of priorities among the criteria for individual DM; the higher the priorities are concentrated on fewer criteria, the lower the entropy. $D$ is the effective number of criteria. For an equal distribution of priorities across all criteria true diversity $D$ equals the number of criteria $N$, and the Shannon entropy $H$ equals $\ln (N)$. For priority given to only one criterion true diversity $D$ is unity, and Shannon entropy $H$ equals $\ln (1)=0$.

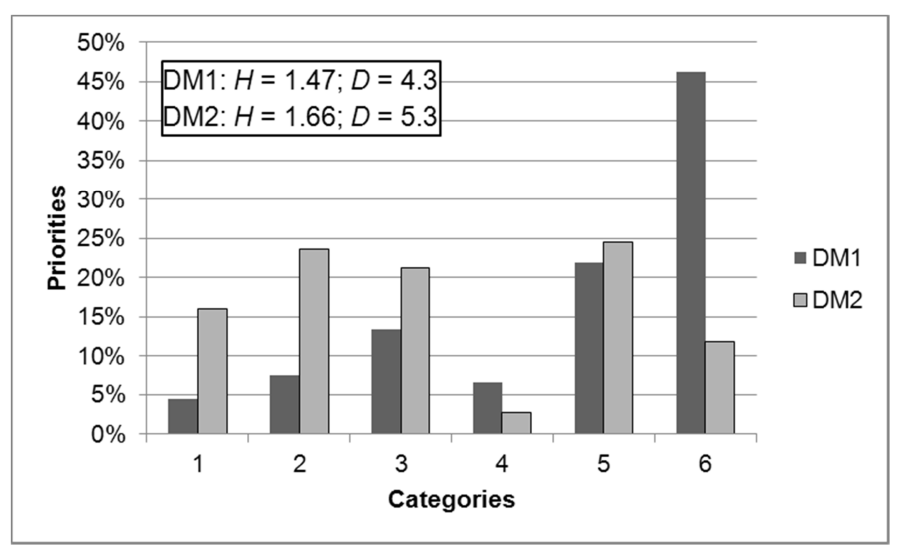

Figure 3. Priority distributions for DM1 and DM2

As an example we take the priorities for two DM of our project 4. As we can see from figure 3 decision maker 1 (DM1) emphasizes criterion 6, the priority distribution among all criteria has a low evenness, resulting in Shannon entropy 1.47 or true diversity of 4.3 (he puts his weights effectively in 4.3 out of the 6 categories). DM2 has a more even distribution of priorities among the criteria with Shannon entropy 1.66 or true diversity of 5.3 .

\subsection{Partitioning Diversity in Alpha- and Beta Diversity}

Using the concept of diversity allows us to partition diversity into two independent components, alpha- and beta diversity (Jost, 2007). Standard alpha Shannon entropy for a group of $K$ decision makers is the average Shannon entropy of all individual DM:

$$
H_{\alpha}=-w_{1} \sum_{i=1}^{N} p_{i 1} \ln p_{i 1}+-w_{2} \sum_{i=1}^{N} p_{i 2} \ln p_{i 2}+\ldots
$$

We will use equal weights with $w_{1}=w_{2}=w_{K}=1 / K$. The number equivalent for (3) is

$$
D_{\alpha}=\exp H_{\alpha}
$$

For the group aggregated priorities we calculate the Shannon gamma diversity $H_{\gamma}$ as

$$
H_{\gamma}=\sum_{i=1}^{K}-\left(w_{1} p_{i 1}+w_{2} p_{i 2}+\ldots\right) \ln \left(w_{1} p_{i 1}+w_{2} p_{i 2}+\ldots\right)
$$

with $w_{1}=w_{2}=w_{K}=1 / K$. The number equivalent (true gamma diversity of order one) is 


$$
D_{\gamma}=\exp H_{\gamma}
$$

Beta diversity $H_{\beta}$ is the difference of $H_{\gamma} \square$ and $H_{\alpha}$ :

$$
H_{\beta}=H_{\gamma}-H_{\alpha}
$$

written as number equivalent (true beta diversity of order one):

$$
D_{\beta}=D_{\gamma} / D_{\alpha}
$$

The maximum of true beta diversity is $N$; the minimum is unity (no variation between DM). Alpha diversity $\left(H_{\alpha}, D_{\alpha}\right)$ measures the average individual decision maker's priority distribution among criteria; beta diversity $\left(H_{\beta}, D_{\beta}\right)$ is a measure of variations of priority distributions among decision makers within the group. A low variation of priority distributions among DM is an indicator for high homogeneity and consensus within the group.

\subsection{Introducing a new homogeneity index as consensus indicator for group decisions}

The reciprocal of true beta diversity (eq. 8) is a simple homogeneity measure (MacArthur, 1965).

$$
M=1 / D_{\beta}=D_{\alpha} / D_{\gamma}
$$

It can be transformed into a relative index of homogeneity in the range from zero to unity:

$$
S=\left(1 / D_{\beta}-D_{\alpha \min } / D_{\gamma \max }\right) /\left(1-D_{\alpha \min } / D_{\gamma \max }\right)
$$

With $D_{\alpha \min }=1$ and $D_{\gamma \max }=N$ we get

$$
S=\left(1 / D_{\beta}-1 / N\right) /(1-1 / N)
$$

The relative index of homogeneity $S$ can be used as a consensus indicator; it is zero, when the priorities of all DM are completely distinct and unity when the priorities of all DM are identical.

Using AHP with an 1 to $M$ scale (standard AHP linear scale: $M=9$ ) the maximum possible priority for one criterion is limited to $M /(N+M-1)$. Alpha entropy is minimal, when the $\mathrm{DM}$ gives full preference to one criterion; Then the priorities - resulting from pair-wise comparisons of $N$ criteria - are $M /(N+M-1)$ for the selected criterion with all other $(N-1)$ priorities equal to $1 /(N+M-1)$. Minimum alpha entropy and maximum gamma entropy for $N$ criteria and $K$ decision makers can be calculated as:

$$
\begin{gathered}
H^{*} \alpha \min =-\frac{M}{N+M-1} \ln \left(\frac{M}{N+M-1}\right)-\frac{N-1}{N+M-1} \ln \frac{1}{N+M-1} . \\
\left.H^{*}{ }_{\gamma \max }=(N-K)\left(-\frac{1}{N+M-1} \ln \frac{1}{N+M-1}\right)-\left(\frac{K+M-1}{N+M-1}\right) \ln \left(\frac{1}{K} \frac{K+M-1}{N+M-1}\right)\right)
\end{gathered}
$$

We use (12) and (13) with (4) and (6) in equation (10) to keep the indicator in the range from 0 to 1 :

$$
S^{*}=\left(1 / D_{\beta}-D^{*}{ }_{\alpha \min } / D^{*}{ }_{\gamma \max }\right) /\left(1-D^{*}{ }_{\alpha \min } / D^{*}{ }_{\gamma \max }\right)
$$

This is our new AHP consensus indicator $S^{*}$ for group decisions.

\subsection{Using beta diversity and the consensus indicator for group decisions in AHP projects}

We can now calculate alpha and beta diversity, the relative homogeneity index $S$ and the AHP consensus indicator $S^{*}$ for our projects using above equations. The results are shown in table 2 . 
Table 2. Shannon diversity $H$ with number equivalent $D$, homogeneity and consensus indicators $S, S^{*}$ for the AHP projects described in this paper

\begin{tabular}{|l|l|l|l|l|l|l|l|l|l|}
\hline No & AHP Projects & Crit & $\mathbf{D M}$ & $\boldsymbol{H}_{\boldsymbol{\alpha}}$ & $\boldsymbol{D}_{\boldsymbol{\alpha}}$ & $\boldsymbol{H}_{\boldsymbol{\beta}}$ & $\boldsymbol{D}_{\boldsymbol{\beta}}$ & $\boldsymbol{S}$ & $\boldsymbol{S}^{*}$ \\
\hline 1 & Service KPIs & 5 & 2 & 1.481 & 4.40 & 0.079 & 1.08 & $85 \%$ & $69 \%$ \\
\hline 2 & Customer Hotline KPIs & 5 & 5 & 1.334 & 3.8 & 0.195 & 1.2 & $79 \%$ & $52 \%$ \\
\hline 3 & Company Performance & & & & & & & & \\
& - Perspective (Level 1) & 3 & 6 & 0.852 & 2.34 & 0.206 & 1.23 & $78 \%$ & $62 \%$ \\
& - Finance (Level 2) & 4 & 6 & 1.156 & 3.18 & 0.161 & 1.18 & $82 \%$ & $69 \%$ \\
& - Productivity (Level 2) & 4 & 6 & 1.136 & 3.12 & 0.079 & 1.08 & $91 \%$ & $84 \%$ \\
\hline 4 & Strategy & & & & & & & & \\
& - Organization & 6 & 9 & 1.564 & 4.78 & 0.156 & 1.17 & $84 \%$ & $70 \%$ \\
& - Business field A & 6 & 14 & 1.557 & 4.75 & 0.218 & 1.24 & $79 \%$ & $61 \%$ \\
& - Business field B & 6 & 12 & 1.561 & 4.76 & 0.205 & 1.23 & $80 \%$ & $63 \%$ \\
\hline 5 & Leadership Values & 7 & 4 & 1.690 & 5.4 & 0,109 & 1.1 & $87 \%$ & $74 \%$ \\
\hline
\end{tabular}

The priorities for project 3, category "Productivity" have the highest consensus $S^{*}$ with $84 \%$; lowest consensus was achieved for project 2, "Customer Hotline KPIs" with 52\%.

\subsection{Clustering}

If we want to gain a deeper insight in the consensus of a group, we use equation (14) for each pair of $\mathrm{DM}(K=2)$ within the group, and arrange the calculated AHP consensus indicator $S^{*}$ in form of a $K \mathrm{x} K$ matrix. Each element of the matrix shows the consensus indicator for a pair of DM. We can then rearrange the sequence of DM to find clusters with high consensus index along the diagonal. Figure 3 shows an example for the category "organization" of project 4.

\begin{tabular}{|c|ccc|cccccc|}
\hline 0 & JS & EF & HG & AW & SL & PN & KG & HM & FS \\
\hline JS & 1 & 0,97 & 0,89 & 0,53 & 0,57 & 0,52 & 0,60 & 0,40 & 0,58 \\
EF & 0,97 & 1 & 0,95 & 0,67 & 0,53 & 0,52 & 0,59 & 0,46 & 0,63 \\
HG & 0,89 & 0,95 & 1 & 0,83 & 0,59 & 0,60 & 0,64 & 0,50 & 0,61 \\
AW & 0,53 & 0,67 & 0,83 & 1 & 0,48 & 0,63 & 0,62 & 0,56 & 0,61 \\
SL & 0,57 & 0,53 & 0,59 & 0,48 & 1 & 0,90 & 0,89 & 0,51 & 0,47 \\
PN & 0,52 & 0,52 & 0,60 & 0,63 & 0,90 & 1 & 0,95 & 0,55 & 0,58 \\
KG & 0,60 & 0,59 & 0,64 & 0,62 & 0,89 & 0,95 & 1 & 0,76 & 0,77 \\
HM & 0,40 & 0,46 & 0,50 & 0,56 & 0,51 & 0,55 & 0,76 & 1 & 0,93 \\
FS & 0,58 & 0,63 & 0,61 & 0,61 & 0,47 & 0,58 & 0,77 & 0,93 & 1 \\
\hline
\end{tabular}

Figure 3: Cluster analysis for decision makers in AHP project 4

Clearly three clusters (sub-groups) of DM with high consensus index can be seen: sub-group 1 (JS, EF, HG), sub-group 2 (SL, PN, KG), and sub-group 3 (HM, FS). From table 2 we see a consensus index $S^{*}$ of $70 \%$ for the whole group. Calculating the consensus indicator for the subgroups we find $94 \%, 92 \%$ and $93 \%$ respectively. Figure 4 shows the similarity of priority distributions from these three subgroups.
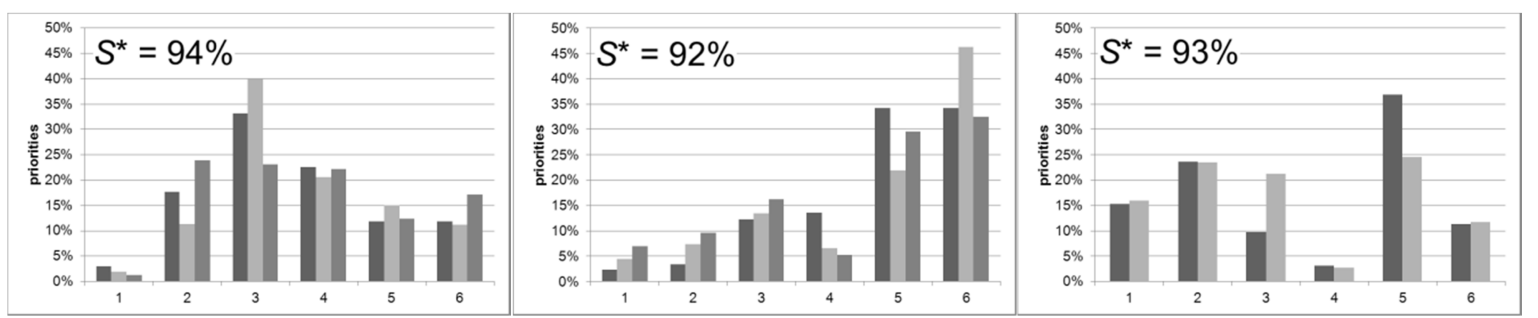

Figure 4. Priorities for DM sub-groups 1 (JS, EF, HG), 2 (SL, PN, KG) and 3 (HM, FS)

Finding clusters of high consensus in the group of DM can be very helpful for further discussions, or even be used to develop new alternatives for a given decision problem. 


\section{Conclusion}

In this paper we described some obstacles in the implementation of AHP as a standard method for multi-criteria decision making in a corporate environment. A flexible AHP spreadsheet template was developed to avoid initial investment for software, and the template was used to conduct several AHP projects.

The most critical issue in the practical implementation of AHP is the requirement of consistent or near consistent matrices. Our projects showed that a strict limit of 0.1 for the consistency ratio CR is not practicable; the median value in the distribution of CR values for all projects was 0.16 . Using the balanced judgment scale improves consistency significantly.

AHP was well accepted for group decisions based on the aggregation of individual judgments, especially for a larger number of decision makers, as they see their inputs reflected. Due to the fact that AHP is based on a mathematical model, the aggregated group results were seen as neutral and objective.

For the further analysis of the consensus within the groups of decision makers we adapted the concept of (bio-) diversity, and introduced the Shannon entropy as a diversity index for the distribution of priorities among criteria. Partitioning in independent alpha and beta components we derived an index for relative homogeneity as consensus indicator. Using this indicator we can cluster the group of decision makers into sub-groups of high consensus, and gain a deeper insight in the judgment and decision process.

\section{REFERENCES}

Alonso, Lamata, (2006). Consistency in the analytic hierarchy process: a new approach. International Journal of Uncertainty, Fuzziness and Knowledge based systems, Vol 14, No 4, 445-459

Aull-Hyde, R. et al. (2006). An experiment on the consistency of aggregated comparison matrices in AHP. European Journal of Operational Research 171, 290 - 295

Bozóki, S., Rapcsák, T. (2007). On Saaty's and Koczkodaj's inconsistencies of pairwise comparison matrices, Journal of Global Optimization, 42(2), 157 - 175

Brunelli, M., Critch A., Fedrizzi M. (2011). A note on the proportionality between some consistency indices in AHP, TUCS Technical Report, No 1012

Goepel, Klaus D. (2013). A new AHP excel template with multiple inputs. The template can be downloaded from http://bpmsg.com

Hill, Mark O. (1973). Diversity and evenness: a unifying notation and its consequences. Ecology $54,427-432$.

Ishisaka, A., Labib, A. (2011). Review of the main developments in the analytic hierarchy process, Expert Systems with Applications 38(11), 14336 - 14345

Jost, Lou (2007). Partitioning diversity into independent alpha and beta components. Ecology 88, 2427-2439

Jost, Lou. (2006). Entropy and diversity. OIKOS 113:2, 363 - 375 
Kaplan, Robert S., Norton David P. (2004). Strategy Maps - Converting intangible assets into tangible Outcomes. Harvard Business School Publishing

Larsen, Ron (2013). Elementary linear algebra. The Pennsylvania State University, The Behrend College ISBN 1133111343

MacArthur, R. (1965). Patterns of Species Diversity. Biol. Rev. 40, 510-533

Saaty, T. L. (2003). Decision making with AHP: Why is the principal eigenvector necessary. European Journal of Operational Research 145, 85-91

Saaty, T. L. (1980). The Analytic Hierarchy Process, Planning, Priority Setting, Resource Allocation. Mc Graw-Hill, NY 1980

Saaty T. L., Vargas, Luis G. (2005). Dispersion of Group Judgments. Proceedings of the International Symposium on the Analytic Hierarchy Process ISAHP 2005, Honolulu, Hawaii

Salo, A., Hämäläinen, R. (1997). On the measurement of preferences in the analytic hierarchy process. Journal of multi-criteria devision analysis, Vol 6, 309-319

Shannon, C. (1948). A mathematical theory of communication. Bell System Tech. J. 27, 379-423, 623-656.

Siraj, Sajid (2011). Preference elicitation from pairwise comparisons in multi-criteria decision making. Dissertation, School of Computer Science, University of Manchester 